\title{
Application of State-Space Model to Exact Time Series Forecasting
}

\author{
Zheng Changjiang ${ }^{1,2}$ \\ ${ }^{1}$ College of Humanities, Shanghai Institute of \\ Technology \\ ${ }^{2}$ An Tai College of Economics \& management, \\ Shanghai Jiao Tong University \\ Shanghai, China
}

Dong yujin

State Grid Electric Vehicle Service Company Shanghai Municipal Electric Power Company Shanghai China

\author{
Cui Youxiang* \\ An Tai College of Economics \& management, Shanghai \\ Jiao Tong University \\ Shanghai, China \\ *Corresponding author \\ E-mail: cuiyouxiang@aliyun.com
}

\author{
Xie Fuji \\ An Tai College of Economics \& management, Shanghai \\ Jiao Tong University \\ Shanghai, China
}

\begin{abstract}
The forecasting method of future values of a time series from current and past values is of considerable practical interest and in important areas of application. In addition to calculating the best forecasts, it is also necessary to specify their accuracy, so that the risks associated with decisions based upon the forecasts may be calculated. Many empirical time series behave as though they had no fixed mean. They exhibit homogeneity in the sense that apart from local level, or perhaps local level and trend, one part of the series behaves much like any other part. Models that describe such homogeneous nonstationary behavior can be obtained by supposing some suitable difference of the process to be stationary. There has been much recent interest in the representation of ARIMA models in the statespace form, for purposes of forecasting, as well as for model specification and maximum likelihood estimation of parameters. In this paper we briefly consider the state-space form of an ARIMA model in this section and discuss its uses in exact finite sample forecasting.
\end{abstract}

Keywords- state space model ; ARIMA; time series analysis; exact forecasting; control engineering

\section{INTRODUCTION}

Many sets of data appear as time series. A time series is a sequence of observations taken sequentially in time.

The forecasting method of future values of a time series from current and past values is of considerable practical interest and in important areas of application.

The use at time $t$ of available observations from a time series to forecast its value at some future time $t+1$ can provide a basis for

(1) Economic and business planning

(2) Production planning

(3) Inventory and production control

(4) Control and optimization of industrial processes.

Forecasts are usually needed over a period known as the lead time, which varies with each problem.

Forecasting is the process of making statements about events whose actual outcomes (typically) have not yet been observed. A commonplace example might be estimation of some variable of interest at some specified future date. Prediction is a similar, but more general term. Both might refer to formal statistical methods employing time series, cross-sectional or longitudinal data, or alternatively to less formal judgmental methods. Usage can differ between areas of application: for example, in hydrology, the terms "forecast" and "forecasting" are sometimes reserved for estimates of values at certain specific future times, while the term "prediction" is used for more general estimates, such as the number of times floods will occur over a long period.

Risk and uncertainty are central to forecasting and prediction; it is generally considered good practice to indicate the degree of uncertainty attaching to forecasts. In any case, the data must be up to date in order for the forecast to be as accurate as possible.

In addition to calculating the best forecasts, it is also necessary to specify their accuracy, so that the risks associated with decisions based upon the forecasts may be calculated. The accuracy of the forecasts may be expressed by calculating probability limits on either side of each forecast. These limits may be calculated for any convenient set of probabilities. To illustrate, Fig .1. shows a time series forecast culminating at time $t$.

Methods for obtaining forecasts and estimating probability limits developed based on the assumption that the time series $z_{t}$ follows a stochastic model named autoregressive integrated moving average (ARIMA) models. 


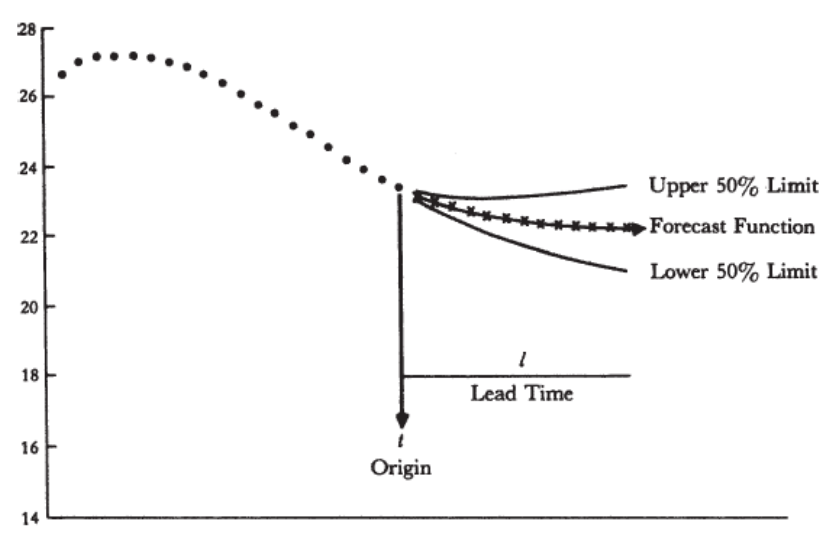

Figure 1. Time series with $50 \%$ probability limits forecast

Time $t$ is the forecast function at origin $t$. The objective is to obtain a forecast function such that the mean square of the deviations between the actual and forecasted values is as small as possible for each lead time $t$.

In control engineering, a state space representation is a mathematical model of a physical system as a set of input, output and state variables related by first-order differential equations. To abstract from the number of inputs, outputs and states, the variables are expressed as vectors. Additionally, if the dynamical system is linear and time invariant, the differential and algebraic equations may be written in matrix form. The state space representation (also known as the "time-domain approach") provides a convenient and compact way to model and analyze systems with multiple inputs and outputs.

\section{ARIMA MODELS IN THE STATE-SPACE FORM}

In statistics and econometrics, and in particular in time series analysis, an autoregressive integrated moving average (ARIMA) model is a generalization of an autoregressive moving average (ARMA) model. These models are fitted to time series data either to better understand the data or to predict future points in the series (forecasting). They are applied in some cases where data show evidence of non-stationarity, where an initial differencing step (corresponding to the "integrated" part of the model) can be applied to remove the non-stationarity.

The model is generally referred to as an $\operatorname{ARIMA}(\mathrm{p}, \mathrm{d}, \mathrm{q})$ model where parameters $\mathrm{p}, \mathrm{d}$, and $\mathrm{q}$ are non-negative integers that refer to the order of the autoregressive, integrated, and moving average parts of the model respectively. ARIMA models form an important part of the Box-Jenkins approach to time-series modelling.

In control engineering, a state space representation is a mathematical model of a physical system as a set of input, output and state variables related by first-order differential equations. To abstract from the number of inputs, outputs and states, the variables are expressed as vectors. Additionally, if the dynamical system is linear and time invariant, the differential and algebraic equations may be written in matrix form. The state space representation (also known as the "time-domain approach") provides a convenient and compact way to model and analyze systems with multiple inputs and outputs. With $p$ inputs and $q$ outputs, we would otherwise have to write down $\mathrm{p} \times \mathrm{q}$ Laplace transforms to encode all the information about a system. Unlike the frequency domain approach, the use of the state space representation is not limited to systems with linear components and zero initial conditions. "State space" refers to the space whose axes are the state variables. The state of the system can be represented as a vector within that space.

There has been much recent interest in the representation of ARIMA models in the state-space form, for purposes of forecasting, as well as for model specification and maximum likelihood estimation of parameters. This paper briefly consider the state-space form of an ARIMA model and discuss its uses in exact finite sample forecasting.

Explicit forms for the general ARIMA model

$$
\varphi(\mathrm{B}) z_{t}=\theta(B) a_{t}
$$

Define the forecasts

$$
\widehat{z_{t}}(j)=E_{t}\left[\left(Z_{t+j}\right)\right], j=0,1,2, \cdots, r
$$

With

$$
\begin{gathered}
r=\max (p+d, q+1 \\
\widehat{z_{t}}(0)=z_{t} \\
\widehat{z_{t}}(j-1)=\widehat{z_{t-1}}(j)+\varphi_{j-1} a_{t} \\
=\sum_{i=1}^{p+d} \varphi_{i} \widehat{z_{t-1}}(j-i)+\varphi_{j-1} a_{t}
\end{gathered}
$$

Define the "state" vector at time $t, Y_{t}$, with $r$ components as

$$
Y_{t}=\left(z_{t}, \widehat{z_{t}}(1), \cdots, \widehat{z_{t}}(r-1)\right)^{\prime}
$$

Then from the relations above we find that the vector $Y_{t}$ satisfies the first-order system of equations:

$Y_{t}=\left[\begin{array}{ccccc}0 & 1 & 0 & \cdots & 0 \\ 1 & 0 & 1 & \cdots & 0 \\ \vdots & \vdots & \vdots & \vdots & \vdots \\ 0 & 0 & \cdots & \cdots & 1 \\ \varphi_{r} & \varphi_{r-1} & \cdots & \cdots & \varphi_{1}\end{array}\right] Y_{t-1}+\left[\begin{array}{c}1 \\ \varphi_{1} \\ \vdots \\ \vdots \\ \varphi_{r-1}\end{array}\right] a_{t}$

Where $\varphi_{i}=0$, if $i>p+d$. So we have

$$
Y_{t}=\Phi Y_{t-1}+\Psi_{a_{t}}
$$

With the observation equation

$$
Z_{t}=z_{t}+N_{t}=[1,0, \cdots, 0] Y_{t}+N_{t}=H Y_{t}+N_{t}
$$

the additional noise $N_{t}$ would be present only if the process $z t$ is observed subject to additional white noise; otherwise, we simply have $z t=\mathbf{H} \boldsymbol{Y}_{t}$. The two Equations above constitute what is a state-space representation of the model, which consists of a state or transition equation (3) and an observation equation (4), and $Y_{t}$ is the state vector. We note that there are many other constructions of the state vector $Y_{t}$ that will give rise to state-space equations 
of the general form of the state-space form of an ARIMA model is not unique. The two equations of the form above, in general, represent a state-space model, with unobservable state vector $Y_{t}$ and observations $Z_{t}$, and can arise in time series settings more general than the context of ARIMA models.

Consider a state-space model of a slightly more general form, with state equation

$$
\begin{aligned}
& Y_{t}=\Phi_{t} Y_{t-1}+a_{t} \\
& Z_{t}=H_{t} Y_{t}+N_{t}
\end{aligned}
$$

Where it is assumed that at and $N_{t}$ are independent white noise processes, at is a vector white noise process with covariance matrix $\sum a$, and $N_{t}$ has variance $\sigma_{N}^{2}$. In this model, the (unobservable) state vector $Y_{t}$ summarizes the state of the dynamic system through time $t$, and the state equation (5) describes the evolution of the dynamic system in time, while the measurement equation (6) indicates that the observations $Z_{t}$ consist of linear combinations of the state variables corrupted by additive white noise. The matrix $\Phi_{t}$ in (5) is an $\mathrm{r} \times r$ transition matrix and $H_{t}$ in (6) is a $1 \times r$ vector, which are allowed to vary with time $t$. Often, in applications these are constant matrices, $\Phi_{t} \equiv$ $\Phi$ and $\mathrm{H}_{\mathrm{t}} \equiv \mathrm{H}$ for all $t$, that do not depend on $t$, as in the state-space form (3) and (4) of the ARIMA model. In this case the system or model is said to be time invariant. The minimal dimension $\mathrm{r}$ of the state vector $Y_{t}$ in a state-space model needs to be sufficiently large so that the dynamics of the system can be represented by the simple Markovian (first-order) structure.

\section{Form OF State-SPace Model AND Steady STATE FOR TIME-INVARIANT MODELS}

One particular alternate form of the general state variable model, referred to as the innovations or prediction error representation, is worth noting. Exact Forecasting in ARIMA Models

Suppose that the transfer function model

$$
\begin{gathered}
Y_{t}^{*}=\hat{Y}_{t \mid t-1} \\
a_{t}^{*}=\hat{a}_{t \mid t-1}=H_{t} \hat{Y}_{t \mid t-1}
\end{gathered}
$$

We get

$$
\begin{gathered}
Y_{t+1}^{*}=\Phi_{t+1} Y_{t}^{*}+\Phi_{t+1} K_{t} a_{t}^{*} \equiv \Phi_{t+1} Y_{t}^{*}+\Psi_{a_{t}}^{*} \\
Z_{t}=H Y_{t}^{*}+a_{t}^{*}
\end{gathered}
$$

Which is also of the general form of a state-space model but with the same white noise process $a_{t}^{*}$ (the onestep-ahead prediction errors) involved in both the transition and observation equations.

In the "stationary case" (i.e., time-invariant and stable case) of the state-space model, where $\Phi_{t} \equiv \Phi$ and $\mathrm{H}_{\mathrm{t}} \equiv \mathrm{H}$ are constant matrices and $\Phi$ has all eigenvalues less than one in absolute value, we can obtain the steady-state form of the innovations representation by setting $Y_{t}^{*}=\mathrm{E}\left[\mathrm{Y}_{\mathrm{t}} \mid \mathrm{Z}_{\mathrm{t}}\right.$ $\left.{ }_{1}, Z_{t-2}, \ldots\right]$, the projection of $Y_{t}$ based on the infinite past of $\left\{Z_{t}\right\}$.

These steady- state results for the time-invariant model case also hold under slightly weaker conditions than stability of the transition matrix such as in the nonstationary random walk plus noise model .Hence in the time-invariant situation, the state variable model can be expressed in the steady-state innovations or prediction error form as

$$
\begin{gathered}
Y_{t+1}^{*}=\Phi Y_{t}^{*}+\Phi K a_{t} \equiv \Phi Y_{t}^{*}+\Psi_{a_{t}}^{*} \\
Z_{t}=H Y_{t}^{*}+a_{t}
\end{gathered}
$$

In particular, for the ARIMA process $\varphi(\mathrm{B}) z_{t}=$ $\theta(B) a_{t}$ with no additional observation error so that $Z_{t}=z_{t}$, a prediction error form the state-space model can be given with state vector

$$
\begin{gathered}
Y_{t+1}^{*}=\left(\hat{z}_{t}(1), \cdots, \hat{z}_{t}\left(r^{*}\right)\right)^{\prime} \\
\Psi^{*}=\left(\varphi_{1}, \cdots, \varphi_{\mathrm{r}^{*}}\right)^{\prime} \\
Z_{t}=\hat{z}_{t-1}(1)+a_{t}
\end{gathered}
$$

And observation equation

At the dimension is $\mathrm{r}^{*}=\max (\mathrm{p}+\mathrm{d}, \mathrm{q})$

\section{EXACT FORECASTING IN ARIMA MODELS}

For ARIMA models, with state space representation (3) and (4)

And

$$
Z_{t}=Z_{t}=H Y_{t}
$$

With

$$
\mathrm{H}=[1,0, \cdots, 0],
$$

The procedure constitutes an alternate method to obtain exact finite sample forecasts, based on data $z_{t}, z_{t-1}, \ldots, z_{1}$, for future values in the ARIMA process, subject to specification of appropriate initial conditions. For stationary zero-mean processes $z_{t}$, the appropriate initial values are a vector of zeros

$$
\widehat{Y}_{0 \mid 0}=0
$$

And

$$
V_{0 \mid 0}=\operatorname{cov}\left[Y_{0}\right] \equiv V_{*}
$$

The covariance matrix of $Y_{0}$, which can easily be determined under stationarity through the definition of $Y_{\mathrm{t}}$. Specifically, since the state vector $Y t$ follows the stationary vector AR(1) model

$$
Y_{t}=\Phi Y_{t-1}+\Psi_{a_{t}}
$$

Its covariance matrix $\mathrm{V}_{*}=\operatorname{cov}\left[\mathrm{Y}_{\mathrm{t}}\right]$ satisfies

$$
V_{*}=\Phi V_{*} \Phi^{\prime}+\sigma_{a}^{2} \Psi \Psi^{\prime}
$$


Which can be readily solved for $\mathrm{V}_{*}$. For nonstationary ARIMA processes, additional assumptions need to be specified.

The forecasts of the ARIMA process $z_{t}$ are obtained recursively as indicated, with 1-step-ahead forecast of the first element of the vector

$$
\hat{z}_{t+l \mid t}=H \hat{Y}_{t+l \mid t}
$$

Where

$$
\hat{Y}_{t}=\Phi \widehat{Y}_{t+l-1 \mid t}
$$

With forecast error variance

$$
v_{t+l \mid t}=H V_{t+l-1 \mid t} H^{\prime}
$$

The "steady-state" values of the 1-step-ahead forecasts $\hat{z}_{t+l \mid t}$ and their forecast error variances $v_{t+l \mid t}$, which are rapidly approached as $t$ increases, will be identical to the expressions given in

$$
V(l)=\sigma_{a}^{2}\left(1+\sum_{j=1}^{l-1} \varphi_{j}^{2}\right.
$$

In particular, for the ARIMA process in state-space form, we can obtain the exact (finite sample) one-stepahead forecasts:

$$
\hat{z}_{t-1}=E\left[z_{t} \mid z_{t-1}, \cdots, z_{1}\right]=H \hat{Y}_{t \mid t-1}
$$

And their error variances

$$
v_{t}=H V_{t \mid t-1} H^{\prime}
$$

This can be particularly useful for evaluation of the likelihood function, based on $\mathrm{n}$ observations $z_{1}, \cdots, z_{n}$ from the ARIMA process, applied to the problem of maximum likelihood estimation of model parameters.

\section{ACKNOWLEDGMENT}

This research was financially supported by the National Science Foundation of China (71373158) and the National Social Sciences Fund of China (12AZD015).

\section{REFERENCES}

[1] Box G E P, Jenkins G M, Reinsel G C. Time series analysis: forecasting and control[M]. Wiley. com, 2013.

[2] http://en.wikipedia.org/wiki/Forecasting
[3] You-xiang C, Jian-xin Y, Feng L. Applying fast Fourier transform (FFT) to statistic quality control[C]//Industrial Engineering and Engineering Management (IEEM), 2010 IEEE International Conference on. IEEE, 2010: 2144-2148. 57670.

[4] http://en.wikipedia.org/wiki/Autoregressive integrate d moving average

[5] http://en.wikipedia.org/wiki/State_space_representati on

[6] Durbin J, Koopman S J. Time series analysis by state space methods[M]. Oxford University Press, 2012.

[7] Bai J, Ghysels E, Wright J H. State space models and MIDAS regressions[J]. Econometric Reviews, 2013, 32(7): 779-813.

[8] Gamerman D, Santos T R, Franco G C. A NON - GAUSSIAN FAMILY OF STATE - SPACE MODELS WITH EXACT MARGINAL LIKELIHOOD[J]. Journal of Time Series Analysis, 2013, 34(6): 625-645.

[9] Škovránek T, Podlubny I, Petráš I. Modeling of the national economies in state-space: A fractional calculus approach[J]. Economic Modelling, 2012, 29(4): 1322-1327.

[10] Loganathan P, Siang H C, Lee H R, et al. Towards forecasting flu dynamics using a regionalized state space model[C]//Advanced Control of Industrial Processes (ADCONIP), 2011 International Symposium on. IEEE, 2011: 175-180.

[11] Croushore D. Forecasting with real-time data vintages[J]. Forecasting Handbook: Oxford University Press. Forthcoming, 2011.

[12] Peng J Y, Aston J A D. The state space models toolbox for MATLAB[J]. Journal of Statistical Software, 2011, 41(6): 1-26.

[13] Francke M K, Koopman S J, De Vos A F. Likelihood functions for state space models with diffuse initial conditions[J]. Journal of Time Series Analysis, 2010, 31(6): 407-414.

[14] Nakajima J, Kunihama T, Omori Y, et al. Generalized extreme value distribution with time-dependence using the AR and MA models in state space form[J]. Computational Statistics \& Data Analysis, 2012, 56(11): 3241-3259.

[15] Doan T. State space methods in RATS[J]. Journal of Statistical Software, 2011, 41(9): 1-16

[16] De Livera A M, Hyndman R J, Snyder R D. Forecasting time series with complex seasonal patterns using exponential smoothing[J]. Journal of the American Statistical Association, 2011, 106(496): 1513-1527.

[17] Ives A R, Dakos V. Detecting dynamical changes in nonlinear time series using locally linear state-space models[J]. Ecosphere, 2012, 3(6): $\operatorname{art} 58$.

[18] DeJong D N, Liesenfeld R, Moura G V, et al. Efficient likelihood evaluation of state-space representations[J]. The Review of Economic Studies, 2013, 80(2): 538-567.

[19] Nielsen L R, Jørgensen E, Højsgaard S. Embedding a state space model into a Markov decision process[J]. Annals of Operations Research, 2011, 190(1): 289-309.

[20] Kumar M, Thenmozhi M. Forecasting stock index returns using ARIMA-SVM, ARIMA-ANN, and ARIMA-random forest hybrid models[J]. International Journal of Banking, Accounting and Finance, 2014, 5(3): 284-308.

[21] Canova F. Bayesian State Space models[J]. 2010.

[22] Youxiang C, Wei H, Jianshe L, et al. Feedforward Control Analytical Model in SPC[J]. 2014. 